included skin manifestations inherent to giardiasis: paleness, cheilitis, hyperkeratosis follicularis punctata and prolonged skin itching. As a result of reflex and toxicoallergic actions of Giardia, a syndrome of chronic endointoxication has prompted emergence of dyskinesia of the gallbladder and sphincter apparatus in $75 \%$ of patients with subsequent inflammation of the gastrointestinal tract (in $48 \%$ of patients). That further increased an antigenic load on their immune systems. SCORAD index in 22 infected children showed moderate severity of atopic dermatitis, whereas 18 patients proved to have a severe form.

Conclusion The analysis has shown that $27 \%$ of patients with atopic dermatitis were infected with giardiasis. These data require a use of a complex approach to the therapy of atopic dermatitis, more thorough examination of children for any pathology of the gastrointestinal tract, particularly, the examination of helminths.

\section{DERMATOLOGIC IMMUNE RECONSTITUTION INFLAMMATORY SYNDROME (IRIS) IN CHILDREN RECEIVING ART FROM A COMMUNITY OUTREACH PROGRAM IN KAMPALA}

doi:10.1136/archdischild-2012-302724.0859

'A Bagenda, 2J Mbabazi, ${ }^{3} \mathrm{M}$ Nalubega-Mboowa. 'International Health Sciences University, Entebbe; ${ }^{2}$ Department of Peadiatrics and Child Health, Mulago Hospital Kampala; ${ }^{3}$ Health Policy and Management, International Health Sciences University, Kampala, Uganda

Background Orikiriiza (2011) demonstrated that dermatological manifestations were the most common IRIS events in children receiving ART. We aimed to find the incidence of dermatologicalIRIS in children receiving ART for at least 12 weeks.

Methodology Retrospective review of medical charts for children who received ART between January 2010-December 2011. Nonadherent and regimen switch children excluded.

Results Total of 110 charts; median age 5.9 years (IOR 9.1months11.9years).70 females (63.6\%). 85children (77.2\%) baseline WHO stage III/IV. All children received septrin. Median time on ART was 24 weeks (IOR 13.2-40.8). Baseline CD4+\% was < 15\% for $77(70 \%)$, $>=15 \%$ for 33 children. Viral load $>399,000$ copies were 76 children $(69.1 \%)$ and $<=399,000$ for 34 children. PPE had the highest incidence (47cases) after ART initiation. 10cases of verrucae planae, 9 Kaposi Sarcoma, Herpes Zoster and Tinea corporis each, 8molluscum contagiosum, 4tinea capitis, 3HSV and 1varicella zoster. Median time to develop PPE was 3weeks (IOR 10.3-50. 1days). Increasing age associated with IRIS; highest between 5-12 years (age correlated with degree of immunosuppression).

Viral load after 3 months; < $1 \log 10$ decrease for 45 children $(40.9 \%),>=1 \log 10$ decrease for 65 children. Children who had $>=25 \mathrm{cells} / \mathrm{ul}$ change in CD4+\% (83 children) carried almost 3-fold risk for dermatologic -IRIS compared to children with $<25$ cells increase (69 Vs 9 cases [O.R 2.9 CI 1.40-11.02, p value 0.004]. No significant increased risk for dermatologic-IRIS based on viral load change.

Conclusion Prevalence of unmasking dermatological-IRIS was high. PPE accounted for highest mucocutaneous IRIS manifestations. Caregivers should be counseled about possible worsening of PPE with ART initiation.

\section{EPIDEMIOLOGICAL FACTORS AND FOOD: WHICH IS THE ROLE IN HELICOBACTER PYLORI RE-INFECTION IN PEDIATRIC AGE?}

doi:10.1136/archdischild-2012-302724.0860

T Sabbi. ASL RMG, Rome, Italy

Background Helicobacter pylori $(\mathrm{Hp})$ infection has been recognized as a cause of chronic gastritis, peptic ulcer, atrophic gastritis and gastric cancer. Its acquisition is related with poor socioeconomic conditions while the relationship of nutrition and $\mathrm{Hp}$ is still a question.

Aim To analyzed if socioeconomic factors and dietary contribute to Hp re-infection in pediatric age.

Patients and methods 150 patients (92 males; age range 5-16 years) with $\mathrm{Hp}$ infection treated and eradicated in the past. 55 patients with $\mathrm{Hp}$ re-infection and 95 patients not re-infected.

We interviewed the children with questionnaire about socioeconomics factors, hygiene, living conditions and their dietary habits.

Results A lower frequency of fermented dairy food, fruits and vegetable consumption was registred among children with $\mathrm{Hp}$ reinfection as compared to not been re-infected.

Among persons with Hp re-infection were noted low socio-economic markers such as croweded living conditions, a large number of siblings and unclean water.

Conclusions Might decrease the risk of Hp re-infection the use of probiotic, vitamin $\mathrm{C}$, antioxidants contained in fruit and vegetables.

Risk factors for Hp re-infection are low socioeconomics factors, hygiene and living conditions.

\section{RSV HOSPITALIZATION IN INFANTS WITH NEUROMUSCULAR DISEASE IN THE CANADIAN REGISTRY OF SYNAGIS ${ }^{\circledR}$ (CARESS) FOLLOWING PROPHYLAXIS (2005-2011)}

doi:10.1136/archdischild-2012-302724.086

'B Paes, ${ }^{2} \mathrm{~A}$ Li, ${ }^{3} \mid$ Mitchell, ${ }^{2} \mathrm{KL}$ Lanctot. 'Paediatrics, McMaster University, Hamilton; ${ }^{2}$ Medical Outcomes and Research in Economics (MORE), Sunnybrook Health Sciences Centre, Toronto, ON; ${ }^{3}$ Paediatrics (Respirology Division), University of Calgary, Calgary, $A B$, Canada

Background and Aims The Canadian Registry of Synagis $\AA$ (CARESS) tracks palivizumab use and respiratory outcomes in highrisk infants, including those with neuromuscular impairments (NMI). We compared respiratory illness (RI) and respiratory syncytial virus positive hospitalization (RSVH) rates in NMI infants versus: 1) those with other underlying medical disorders (MD) and 2) those prophylaxed for standard indications (SD).

Methods A prospective, observational registry of infants from 30 Canadian sites who received $\geq 1$ dose of palivizumab during the 2005-2011 RSV seasons. Utilization and RI events were collected monthly throughout each season.

Results 10452 infants were recruited (NMI: 118, 1.1\%; MD: 1443, 13.8\%; SD: 8891, 85.1\%). There were statistically significant group differences $(p<0.05)$ in: enrolment weight and age, gestational age, birth weight, proportions of: Caucasians, daycare attendance, smoking exposure, siblings, multiple birth, $>5$ individuals in the household, and history of atopy. NMI infants tended to have a less complex neonatal course. Compliance was similar across the three groups. The NMI group had higher RI hospitalization rates than $\mathrm{MD}$ or $\mathrm{SD}(17.8 \%$ versus $9.6 \%$ and $5.8 \%, \mathrm{p}<0.0005)$, as well as RSVH (5.62\% versus $1.98 \%$ and $1.49 \%, \mathrm{p}<0.0005)$. A Cox proportional hazard analysis showed that having NMI increased the risk of first RSVH compared to infants in the SD group (hazard ratio $=4.47$, 95\% CI 1.96-10.18, p<0.0005).

Conclusions NMI infants comprise a very high risk cohort for RI and RSV-related hospitalization and should be considered for palivizumab prophylaxis to reduce incurred morbidities as recommended by the American Academy of Pediatrics and other international advisory bodies. THE CANADIAN REGISTRY OF SYNAGIS ${ }^{\circledR}$ (CARESS) FOLLOWING PROPHYLAXIS (2005-2011)

doi:10.1136/archdischild-2012-302724.0862 
'B Paes, ${ }^{2} \mathrm{~A}$ Li, ${ }^{3}$ I Mitchell, ${ }^{2} \mathrm{KL}$ Lanctot. ${ }^{1}$ Paediatrics, McMaster University, Hamilton; ${ }^{2}$ Medical Outcomes and Research in Economics (MORE), Sunnybrook Health Sciences Centre, Toronto, ON; ${ }^{3}$ Paediatrics (Division of Respirology), University of Calgary, Calgary, $A B$, Canada

Background and Aims Aboriginal infants are at substantially higher risk for respiratory illness (RI) and respiratory syncytial virus (RSV) infection and hospitalization compared to non-Aboriginal infants. The purpose of the present study is to compare the hospitalization rates for RI events and RSV infection in Aboriginal infants versus non-Aboriginal infants in the CARESS database.

Methods A prospective, observational registry of infants from 30 Canadian sites who received $\geq 1$ dose of palivizumab during the 2005-2011 RSV seasons. Utilization and hospitalization outcomes were collected monthly throughout respective RSV seasons.

Results 10,452 infants were recruited (318 Aboriginal; 10,134 nonAboriginal). A greater proportion of Aboriginal infants had factors that increased their risk of RSV infection ( $p<0.05)$ : having siblings, being a multiple birth, exposure to smoking, and $>5$ individuals in the household. Aboriginal infants were less compliant with treatment $(p<0.05)$ whether calculated by injection intervals or by expected number of injections during the season. Aboriginal infants had a significantly higher RI hospitalization rate (13.2\% versus $6.2 \%, \mathrm{p}<0.005)$, but only a trend towards a higher RSV-positive hospitalization rate (2.64\% versus $1.57 \%, \mathrm{p}=0.059)$. A Cox proportional hazards analysis restricted to Aboriginal infants found the risk of RSV-positive hospitalization was higher among non-compliant than compliant infants (hazard ratio $=9.2,95 \%$ CI 1.1-76.7, $\mathrm{p}=0.04$ ). Conclusions This study confirms that several demographic and environmental factors that are prominent in enhancing the risk of both RSV infection and overall RI hospitalizations are at play in Aboriginal infants. Ensuring compliance with prophylaxis will likely reduce RSV hospitalization rates in this vulnerable population.

\section{3 \\ IS CEREBRAL MALARIA OVER DIAGNOSED IN CHILDREN IN SUDAN?}

doi:10.1136/archdischild-2012-302724.0863

${ }^{1}$ EO El-Amin, ${ }^{2,3}$ OE Elamin, ${ }^{2} \mathrm{Ml}$ Haj El Bashir, ${ }^{4}$ I Abdulrahman, ${ }^{2} \mathrm{ME}$ El-Amin. ${ }^{1}$ Paediatrics, Faculty of Medicine, The National Ribat University; ${ }^{2}$ Paediatrics, Ministry of Health, ${ }^{3}$ Sudan Medical Speciaization Board; ${ }^{4}$ Pathology, The Children's Memorial Health Institute in Warsow National Ribat University, Khartoum, Sudan

Background WHO defined cerebral malaria (CM) in 1990 as a clinical syndrome of Plasmodium falciparum infection with unrousable coma not attributable to another cause. This has been broaden by adding altered consciousness, severe anemia, and respiratory distress without laboratory confirmation in order to curtail mrtality in children. This has resulted in overdiagnosis and overlooking other serious alternatives plus overburdening the scarce resources.

Aims To analyze the situation in Sudan by studying children admitted with clinical CM and do all the possible diagnostic work up in order to reach definitive diagnosis

Patients and methods Patients belonged t the main hospitals in the capital Khartoum admitting to well organized emergency departments. Clinical and laboratory data were collected from children over 1 month of age admitted with clinical CM between April and November 2011. Patients were investigated for CM, acute bacterial meningitis (ABM) and Herpes encephalitis (HE).

Results One hundred and four children fulfilled the study criteria. CM was clinically diagnosed in 38 patients but only 5 were pure CM. Sixty three were suspected for ABM but 15 were confirmed cases. HE was defenitively diagnosed in only one case. There were 5 cases of mixed infection and the rest were unknown and presumed encephalitis due to viruses other than Herpes simplex.
Conclusion CM was clinically over-diagnosed in our study. It is advisable to do all the necessary investigations, particularly a thoruogh blood film examinatiom, beforediagnosing CM. It is recommended to study cases that resemble CM for more detailed viruses disease.

\section{ASSOCIATION OF FUNGAL INFECTION AND INCREASED MORTALITY IN CHILDREN WITH CHRONIC GRANULOMATOUS DISEASE}

doi:10.1136/archdischild-2012-302724.0864

'S Bassiri-Jahromi, ${ }^{2 A}$ Doostkam. 'Medical Mycology Department, Pasteur Institute, Tehran; ${ }^{2}$ Shiraz University of Medical Sciences, Education Development Center, Shiraz, Iran

Background The aim of this study was to determine the incidence of fungal infections, identify the most common fungal pathogens, and determine the risk factors associated with fungal infections and mortality in children with chronic granulomatous disease (CGD)

Material and Methods All of the patients were suspected to fungal infections. The data was gathered from the medical records of all children as having CGD. The diagnostic of fungal infections were confirmed by histopathology and direct preparation, culture techniques, histopathology of surgical biopsies, and radiological examination of the affected site.

Results We evaluated twelve cases of chronic granulomatosis Patients that they are susceptible to recurrent, sever infections. Children consisted of 7 males and 5 females. The median age of patients at the time of the study was 11.66 years ( 3 to18). Neutrophil oxidative burst were absent $(\mathrm{NBT}=0)$ in all patients. Fungal infections were confirmed in five patients (41/7\%) by histology and mycological methods. The most common isolated fungi in this study were Aspergillus sp. Out of 5 cases of fungal infections identified, tree were Aspergillus species, and two Fusarium species. The most common manifestations of CGD due to fungal infections were osteomyelitis (42.8\%), pulmonary infections $(28.6 \%)$, lymphadenopathy $(14.3 \%)$ and skin involvement (14.3\%) during their illness.

Conclusion Invasive fungal infections are a frequent and lifethreatening complication in CGD patients. The lungs and skeletal, were the most commonly affected organ; however, lymphatic, and skin involvement have also been described. Our present study showed that fusariosisis also is a threat to CGD patients.

\section{INTRANASAL MIDAZOLAM AND KETAMINE FOR GASTRIC ASPIRATES IN CHILDREN EVALUATED FOR SUSPECTED TUBERCULOSIS}

doi:10.1136/archdischild-2012-302724.0865

A Chiaretti, P Valentini, F Pierri, C Fantacci, 0 Genovese, D Buonsenso. Catholic University of the Sacred Heart, Rome, Italy

Background and Aims To confirm the diagnosis of pulmonary tuberculosis in children sequential gastric lavages are recommended. Limitations of gastric lavage include the need for an overnight fast, repeated specimens, and low sensitivity. Moreover, the procedure is very unpleasant for children, parents, and health workers; so sedation may be recommended. We evaluate the safety and efficacy of intranasal administration of midazolam and ketamine in uncooperative children undergoing gastric aspirates to diagnose pulmonary tuberculosis.

Methods We studied 11 children with suspected tuberculosis. Gastric lavages were done on three consecutive days after intranasal administration of midazolam $(0.5 \mathrm{mg} / \mathrm{kg})$ and ketamine $(2 \mathrm{mg} / \mathrm{kg})$ by a mucosal atomizer device. Pain score was assessed by the MOPS score, ranging from 0 to 10 (the higher the score the greater the pain experienced for the child). Gastric specimens underwent polymerase 